\title{
Correspondence
}

\section{Pain and rheumatoid arthritis}

SIR, Gibson and Clark $^{1}$ have indicated that rheumatologists may underestimate their patients' desire for pain relief. In their survey $47 \%$ of a random sample of patients with rheumatoid arthritis ranked pain relief as the most desirable objective of treatment. We also have been impressed by the knowledge that pain is the major symptom of our patients. We surveyed 250 patients and asked them to rank the importance of treatment related to the relief of four symptoms: pain, stiffness, joint swelling. and disability. The order in which the symptoms were presented to the patients was varied. One hundred and twenty two patients were suffering from rheumatoid arthritis, 53 from degenerative joint disease, and the remainder from a variety of other rheumatic diseases. $66 \%$ of the rheumatoid patients and $75 \%$ of the patients with degenerative joint disease ranked pain as the most important symptom to be treated. Only $22 \%$ of rheumatoid patients and $15 \%$ of patients with degenerative joint disease ranked disability as the most important symptom. Stiffness was ranked third by both groups, with $12 \%$ and $6 \%$ respectively. The patients with rheumatoid arthritis who considered disability to be all important were, in general, patients with end stage disease who had rheumatoid deformities without active synovitis. One wonders therefore whether the relatively small proportion of patients in Gibson and Clark's survey who ranked pain relief important may have included a large proportion with end stage disease. Although the patients with degenerative joint disease considered similar priorities of treatment, they tended to have greater difficulty in differentiating the symptoms and appeared less dogmatic.

The message however remains clear: the success of treatment in the rheumatic diseases needs to be assessed by the relief of the patients' most important symptom - pain.

Rheumatism Research Unit, 36 Clarendon Road,

Leeds

\section{Reference}

1 Gibson T, Clark B. Use of simple analgesics in rheumatoid arthritis. Ann Rheum Dis 1985; 44: 27-9.

\section{Frozen shoulder}

SIR, I read with considerable interest the recent article by Binder and associates ${ }^{1}$ in which patients with a frozen shoulder were assessed by arthrography and radionuclide studies. I have several questions and comments regarding the application of the first of these two modalities.
The authors report that their results disagreed with those contained in previous articles in that only half of the patients with adhesive capsulitis showed characteristic arthrographic abnormalities. These data are of concern to those of us who commonly perform arthrography in an attempt to diagnose and categorise the severity of capsular fibrosis. Perhaps an explanation for the seemingly low incidence of positive findings is related to arthrographic technique. Binder and associates state that a doublecontrast examination was used, accomplished by injecting a combination of $5 \mathrm{ml}$ of positive contrast material (Conray 280 ) and $5-15 \mathrm{ml}$ of air. The usefulness of the doublecontrast technique has been well described in previous articles and is most applicable to the evaluation of patients with suspected rotator cuff disruption. ${ }^{23}$ It is not the procedure of choice in the diagnosis of adhesive capsulitis, as early leakage of air and contrast material from the subcapsularis bursa and biceps tendon sheath occurs, a phenomenon that obscures the characteristic arthrographic alterations of adhesive capsulitis. In fact even those who are the most enthusiastic advocates of the double-contrast shoulder arthrogram indicate that adhesive capsulitis is better evaluated by a single positive-contrast examination. ${ }^{3}$ Furthermore, with this latter type of examination, slow distension of the glenohumeral joint with a mixture of contrast material and saline at the time of arthrography, after the diagnosis of adhesive capsulitis has been made, may provide excellent, although temporary, relief of the clinical manifestations of the frozen shoulder. ${ }^{4}$

These comments would indicate that meaningful data about the use of arthrography in the patient with adhesive capsulitis cannot be obtained if double-contrast arthrography of the shoulder has been employed. However, although Binder and associates state that such a technique was used in their study Figs 1 and 2 in their article appear to illustrate the single-contrast examination, as no air can be identified in the images. Perhaps, the authors could explain this discrepancy.

University of California, San Diego,

DONALD RESNICK Chief, Radiology Service,

Veterans Administration Medical Center,

$3350 \mathrm{La}$ Jolla Village Drive,

San Diego, California 92161.

\section{References}

1 Binder A I, Bulgen D Y, Hazleman B L. Tudor J, Wraight P. Frozen shoulder: an arthrographic and radionuclear scan assessment. Ann Rheum Dis 1984: 43: 365-9.

2 Ghelman B, Goldman A B. The double-contrast shoulder arthrogram: evaluation of rotator cuff tears. Radiology 1977: 124: $251-4$.

3 Goldman A B. Ghelman B. The double-contrast shoulder arthrogram. A review of 158 studies. Radiology 1978; 127: 655-63. 